\title{
A meta-analysis of risk factors for depression in adults and children after natural disasters
}

\author{
Bihan Tang ${ }^{\dagger}$, Xu Liu $^{\dagger}$, Yuan Liu, Chen Xue and Lulu Zhang ${ }^{*}$
}

\begin{abstract}
Background: A number of studies have shown a range of negative psychological symptoms (e.g. depression) after exposure to natural disasters. The aim of this study was to determine risk factors for depression in both children and adults who have survived natural disasters.

Methods: Four electronic databases (PubMed, Embase, Web of Science, and Psychlnfo) were used to search for observational studies (case-control, cross-sectional, and cohort studies) about depression following natural disasters. The literature search, study selection, and data extraction were conducted independently by two authors. Thirty-one articles were included in the study, of which twenty included adult participants and eleven included child participants. Summary estimates were obtained using random-effects models. Subgroup analysis, sensitivity analysis, and publication bias tests were performed on the data.

Results: The prevalence of depression after natural disasters ranged from $5.8 \%$ to $54.0 \%$ in adults and from $7.5 \%$ to $44.8 \%$ in children. We found a number of risk factors for depression after exposure to natural disasters. For adults, the significant predictors were being female ;not married;holding religious beliefs; having poor education; prior trauma; experiencing fear, injury, or bereavement during the disaster; or losing employment or property, suffering house damage as a result of the disaster. For children, the significant predictors were prior trauma; being trapped during the disaster; experiencing injury, fear, or bereavement during the disaster; witnessing injury/death during the disaster; or having poor social support.
\end{abstract}

Conclusions: The current analysis provides evidence of risk factors for depression in survivors of natural disasters. Further research is necessary to design interventions to improve the mental health of survivors of natural disasters.

Keywords: Depression, Risk factors, Children, Adults, Natural disasters

\section{Background}

Natural disasters have a devastating impact on affected regions and their populations, often causing death and serious personal injury. Survivors of the disaster often experience mental health problems in the aftermath. One of the most common mental health problems for survivors of natural disasters is depression [1]. Depression is a risk factor for a range of diseases and poor health outcomes [2]. It is important to understand what factors may give rise to depression following a natural disaster. Studies investigating the prevalence of depression after such events indicate that the percentage of people that experience depression ranges from $4.9 \%$ to $54 \%$ [3-33]. Such variability

\footnotetext{
* Correspondence: zllsmmu@126.com

${ }^{\dagger}$ Equal contributors

Institute of Military Health Management, Second Military Medical University, 800 Xiangyin Rd, Shanghai 200433, China
}

can only be partially explained by the differences among these studies in diagnostic tools, sampling frames, and study design [20]. Differences in basic characteristics, trauma characteristics, and post-trauma characteristics may explain the large variation in depression rates between studies.

The onset of depression following natural disasters has been studied for more than 20 years. Studies have demonstrated that sometimes there is a delay in the onset of depression in both children and adults. Depression can be experienced weeks or months after the natural disaster, and in some cases persists for years [34,35]. Several meta-analyses have been carried out on risk factors for depression in different populations. These have focused on populations affected by humanitarian emergencies, such as refugees, internally displaced persons, populations affected 
by mass conflict/displacement, and populations affected by deadly diseases such as cancer and stroke [36-39]. To date, however, there has been no meta-analysis of risk factors for depression in populations specifically affected by natural disasters.

Understanding risk factors for experiencing depression after natural disasters can help clinicians provide more tailored treatments to reduce symptoms and aid post-disaster recovery. This study investigates the determinants of depression in survivors of natural disasters using a systematic meta-analysis of observational studies.

\section{Methods}

Methods and reporting were in accordance with MOOSE (meta-analysis of observation studies in epidemiology) guidelines (The Additional file 1) [40].

\section{Data sources and search strategy}

Observational studies (case-control, cross-sectional, and cohort studies) on risk factors for depression after natural disasters published in English were included in our meta-analysis, irrespective of publication status and article type. Two investigators (B. T. and X. L.) conducted a systematic literature search using the electronic databases PubMed (from 1965 to April 2014), Embase (from 1965 to April 2014), Web of Science (from 1986 to April 2014) and PsychInfo (from 1990 to June 2013). As describing in Additional file 2: Table $\mathrm{S} 1$, we used MeSH terms ("mental disorders", "Depression", "Depressive Disorder", "Earthquakes", "Tsunamis", "Floods", "Cyclonic Storms", "Volcanic Eruptions", "Tornadoes", "Landslides", "Droughts") and free texts for the PubMed search, Emtree ("depression", "mental disease", "earthquake", "tsunami", "flooding", "hurricane", "landslide", "drought", "natural disaster") for the Embase search, and free texts for Web of Science and PsycINFO search. Additionally, manual searches of references cited in all relevant original and review articles were conducted. If there were some full texts unavailable in the databases, we attempted to obtain information from the authors by email.

\section{Selection and exclusion criteria}

In order for studies to be eligible for inclusion in the meta-analysis, they had to fulfil the following criteria: (1) was an epidemiological investigation of risk factors for depression after natural disasters; (2) reported the relative risks (RRs) or odds ratios (ORs) and corresponding 95\% confidence intervals (CIs) for risk factors in the development of depression; (3) included risk factors for depression after the natural disasters which we studied; and (4) included a study sample of children, adults, or both. The exclusion criteria were as follows: (1) used depression score as a variable, but did not obtain ORs or RRs; (2) were not published in English; and (3) focused on participant groups known to be susceptible to depression (i.e. pregnant women and people suffering from mental illness). Studies in which most of the study sample was less than 18 years old were classified as child studies; otherwise, they were classified as adult studies. If more than one article reported data from the same population, then the most recent and complete article was included in our meta-analysis. Study selection and application of inclusion criteria were carried out independently by the two investigators who conducted the literature search (B.T. and X.L.).

\section{Data extraction and quality assessment}

Data extraction was independently performed by two investigators (B.T. and Y.L.). The following information was extracted from each eligible study: first author's surname, year of publication, study location, disaster type, study design, study population, diagnosis of depression, sample size, depression prevalence, interval between research and the date of the natural disaster, age and gender of participants, estimated effect size (OR/RR), corresponding 95\% $\mathrm{CI}$, and covariates adjusted in the statistical analysis. For studies that reported several multivariable-adjusted effect estimates, we selected the one that adjusted for more potential confounding variables.

Quality assessments were conducted independently by two investigators (B.T. and X.L.) using an 11-item instrument recommended by the Agency for Healthcare Research and Quality (AHRQ) for cross-sectional studies [41] and the 9-star Newcastle-Ottawa Scale (NOS) [42] for case-control and cohort studies. Studies that recorded a score of seven stars or more were considered high quality. The quality assessment of the original articles was reexamined and adjudicated independently by an additional investigator (L.Z.).

\section{Search results and characteristics of studies}

The Additional file 3: Figure S1 shows the complete selection process. Up to April 2014, 5,967 records were retrieved by our search strategy. We excluded 5,788 articles after reading the titles and abstracts, and retained 179 articles for further evaluation by reading the full texts. The Additional file 4: Table S2 shows the 148 excluded articles and detailed reasons for exclusion after full-text reading. Finally, we selected 31 full-text articles about risk factors for depression after natural disasters for our meta-analysis [3-33]. There were 21 articles that focused on earthquakes, 7 on hurricanes/tornadoes/typhoons, 2 on tsunamis, and 1 on floods. Twenty studies investigated the association between risk factors and depression in adult survivors of natural disasters, totalling 4,548 depression cases out of 28,217 participants. Eleven studies investigated the association between risk factors and depression in child 
survivors of natural disasters, totalling 2,816 depression cases out of 12,890 participants. Table 1 shows the general characteristics of the 31 studies included in the analysis.

\section{Classification of risk factors}

According to previously published studies $[14,43,44]$, risk factors for depression among children and adults after natural disasters were divided into three categories: basic characteristics (including age, gender, education, marital status, religious beliefs, prior trauma and prior physical illness), trauma characteristics (including being trapped; experiencing fear, injury, or bereavement, e.g. losing close friends or family members; or witnessing injury/death as a result of the natural disasters), and post-trauma characteristics (including amount of social support, employment, loss of property, and house damage).

\section{Statistical analysis}

We examined risk factors for depression after natural disasters by looking at the adjusted ORs and 95\% CIs reported in each study. A random-effects model [45], which assumes that the true underlying effect varies among included studies, was used to estimate the pooled RRs with 95\% CIs. Heterogeneity between studies was evaluated by the $X^{2}$ test and I2 statistic [46]. The probability of publication bias was assessed with the Egger's regression test [47]. Subgroup analyses and sensitivity analyses were performed after excluding lowquality studies, unadjusted results, research on the Wenchuan earthquake, and studies long after the disaster onset ( $>6$ months). Twelve research articles on the Wenchuan earthquake, which made up nearly half (38.7\%) of the incorporated articles, were excluded to explore whether this would make a significant change to the results. We also excluded studies that were implemented a long time after the onset of the disaster ( $>6$ months). Having a long period between the research and the natural disaster raises the possibility that participants may have been exposed to subsequent traumatic events in addition to the natural disaster, which may confound the interpretation of results. If risk factors were multi-categorical variables, we used an OR of the highest versus lowest category (such as age in some studies, education level, scare, house damage, loss of property, and social support). If risk factors were continuous variables (such as age in some studies), they were excluded to avoid inaccuracy because it is not appropriate to combine the ORs from continuous and segmental data.

Stata Version 12.0 software (Stata Corp, College Station, TX) was used for all analyses and all statistical tests were two-sided. A value of $\mathrm{p}<0.05$ was considered an indication of statistical significance.

\section{Results}

\section{Risk factors for depression in adults}

The prevalence of depression in adults after natural disasters ranged from $5.8 \%$ to $54.0 \%$. The risk factors for depression after natural disasters in adults are presented in Table 2 and Figure 1. Regarding the basic characteristics of survivors, we found that being female, having a low-level education, not being married, following a religion, and prior trauma were significantly associated with depression after natural disasters, with pooled ORs of 1.57 (95\% CI, 1.391.79), 1.70 (95\% CI, 1.29-2.23), 1.43 (95\% CI, 1.03-1.98), 1.37 (95\% CI, 1.02-1.86), and 2.26 (95\% CI, 1.34-3.81), respectively. However, heterogeneity was found for education (I2 $=72.7 \%, \mathrm{p}<0.001)$, marriage $(\mathrm{I} 2=73.7 \%, \mathrm{p}<0.001)$, prior trauma ( $\mathrm{I} 2=67.9 \%, \mathrm{p}=0.025)$, and prior physical illness $(\mathrm{I} 2=81.3 \%, \mathrm{p}<0.001)$. The subgroup and sensitivity analyses showed inconsistencies in the results for marriage, religion, prior trauma, and prior physical illness, which should be interpreted with caution because of potential bias. In addition, we found a publication bias for education (Egger's test $\mathrm{p}=0.008$ ) and marriage (Egger's test $\mathrm{p}=0.030$ ). Thus, after adjusting for publication bias, the OR was 1.31 (95\% CI, 0.98-1.76) for education and 1.43 (95\% CI, 1.031.98) for marriage.

With regard to the trauma characteristics of survivors, people who experienced fear, injury, or bereavement during a natural disaster were more likely to suffer from depression, with pooled ORs of 1.58 (95\% CI, 1.37-1.83), 1.69 (95\% CI, 1.39-2.06), and 1.51 (95\% CI, 1.22-1.86) with no heterogeneity or publication bias. All the results were consistent according to the subgroup and sensitivity analyses.

Finally, analysis of the post-trauma characteristics of survivors showed that unemployment (OR 1.55, 95\% CI, $1.02-2.37$ ), loss of property (OR $1.66,95 \% \mathrm{CI}, 1.11-$ 2.47 ), and house damage (OR 1.40, 95\% CI, 1.00-1.88) were related to depression. However, heterogeneity was found for unemployment ( $22=89.4 \%, \mathrm{p}<0.001$ ), loss of property $(\mathrm{I} 2=74.7 \%, \mathrm{p}=0.001)$, and house damage $(\mathrm{I} 2=$ $51.3 \%, \mathrm{p}<0.045)$, suggesting that findings on these variables show inconsistencies.

\section{Risk factors for depression in children}

The prevalence of depression in children after natural disasters ranged from $7.5 \%$ to $44.8 \%$. The risk factors for depression after natural disasters in children are presented in Table 3 and Figure 2. Regarding the basic characteristics of survivors, the pooled analysis had shown that only prior trauma was associated with risk of depression (OR 1.73 95\% CI 1.16-2.58) with high heterogeneity ( $\mathrm{I} 2=86.6 \%, \mathrm{p}=0.001$ ). However, after excluding the Wenchuan earthquake data, prior trauma was associated with the risk of depression, but this relationship was not significant. In addition, gender and age were not related to risk of depression. 
Table 1 General characteristic of the included studies with regard to risk factors for depression after natural disasters

\begin{tabular}{|c|c|c|c|c|c|c|c|c|c|c|c|c|c|}
\hline Id & Author & Year & Country & Disaster type & $\begin{array}{l}\text { Study } \\
\text { design }\end{array}$ & Population & Diagnosis of depression & $\begin{array}{l}\text { Sample } \\
\text { size }\end{array}$ & $\begin{array}{l}\text { Depression } \\
\text { prevalence }\end{array}$ & $\begin{array}{l}\text { Interval between } \\
\text { research and } \\
\text { disaster }\end{array}$ & $\begin{array}{l}\text { Male } \\
\%\end{array}$ & Age & Quality \\
\hline 1 & Guo & 2014 & China & Wenchuan earthquake & cross section & adults & $\begin{array}{c}\text { Center for } \\
\text { Epidemiologic Studies } \\
\text { Depression Scale }\end{array}$ & 633 & $22.9 \%$ & 6 months & $41.7 \%$ & $31-86$ & 7 \\
\hline 2 & Nillni & 2013 & Armenia & Hurricane Katrina & cross section & adults & $\begin{array}{l}\text { Patient Health } \\
\text { Questionnaire-9 }\end{array}$ & 810 & $11.7 \%$ & 18 month & $47.8 \%$ & $>=18$ & 6 \\
\hline 3 & Boscarino & 2013 & US & Hurricane Sandy & cross section & adults & $\begin{array}{l}\text { A major depressive } \\
\text { disorder scale }\end{array}$ & 200 & $6.0 \%$ & 6 months & $35 \%$ & $>=18$ & 6 \\
\hline 4 & Zhou & 2013 & China & Wenchuan earthquake & cross section & adults & $\begin{array}{l}\text { Structured Clinical } \\
\text { Interview for DSM-IV- } \\
\text { TR axis I disorder }\end{array}$ & 14207 & $11.0 \%$ & 6 months & $48.42 \%$ & $>15$ & 6 \\
\hline 5 & Gigantesco & 2013 & Italy & L'Aquila earthquake & cross section & adults & $\begin{array}{l}\text { Patient Health } \\
\text { Questionnaire } 8\end{array}$ & 895 & $5.8 \%$ & 14-19 months & $49.2 \%$ & $18-69$ & 7 \\
\hline 6 & Cheng & 2013 & China & Wenchuan earthquake & cross section & adults & $\begin{array}{l}\text { Structured Clinical } \\
\text { Interview for DSM-IV } \\
\text { Axis I Disorders }\end{array}$ & 182 & $48.9 \%$ & 12 months & $34.8 \%$ & $>=18$ & 6 \\
\hline 7 & Cerda & 2013 & Haiti & Haiti earthquake & cross section & adults & $\begin{array}{l}\text { Patient Health } \\
\text { Questionnaire } 9\end{array}$ & 1315 & $28.3 \%$ & 2-4 months & $28.9 \%$ & $>=18$ & 6 \\
\hline 8 & $\mathrm{Vu}$ & 2012 & Armenia & Hurricane Katrina & cohort & adults & $\begin{array}{l}\text { 18-item Vietnamese } \\
\text { depression scale }\end{array}$ & 128 & $8.5 \%$ & 12 month & $66.4 \%$ & $28-52$ & 7 \\
\hline 9 & Zhang & 2012 & China & Yushu earthquake & cross section & adults & $\begin{array}{l}\text { Hopkins Symptoms } \\
\text { Checklist-25 }\end{array}$ & 505 & $38.6 \%$ & 3-4 months & $53.5 \%$ & $16-87$ & 7 \\
\hline 10 & Zhang & 2012 & China & Wenchuan earthquake & cross section & the Elderly & $\begin{array}{l}\text { Hopkins Symptoms } \\
\text { Checklist-25 }\end{array}$ & 274 & $35.2 \%$ & 14 months & $38 \%$ & $60-98$ & 9 \\
\hline 11 & Zhang & 2011 & China & Wenchuan earthquake & cross section & adults & $\begin{array}{l}\text { Hopkins Symptoms } \\
\text { Checklist-25 }\end{array}$ & 1181 & $49.6 \%$ & 1 year & 37.3 & $16-98$ & 9 \\
\hline 12 & Tracy & 2011 & America & Hurricane lke & cross section & adults & $\begin{array}{l}\text { Patient Health } \\
\text { Questionnaire-9 }\end{array}$ & 658 & $4.9 \%$ & 2-5 months & $49.4 \%$ & $>=18$ & 7 \\
\hline 13 & Paranjothy & 2011 & England & UK floods & cross section & adults & $\begin{array}{l}\text { Patient Health } \\
\text { Questionnaire-9 }\end{array}$ & 2113 & $13.3 \%$ & $\begin{array}{l}3 \text { months } \\
\text { and } 6 \text { months }\end{array}$ & $48 \%$ & $16-96$ & 6 \\
\hline 14 & Anwar & 2011 & Pakistan & Pakistan earthquake & cross section & women & $\begin{array}{l}\text { Hopkins Symptom } \\
\text { Checklist-25 }\end{array}$ & 387 & $54.0 \%$ & 4 years & - & $15-49$ & 7 \\
\hline 15 & Amstadter & 2009 & Vietnam & typhoon Xangsane & cross section & adults & $\begin{array}{l}\text { Structured Clinical } \\
\text { Interview for DSM-IV }\end{array}$ & 798 & $5.9 \%$ & 3 months & - & $18-96$ & 6 \\
\hline 16 & Van Griensven & 2006 & Thailand & Thailand Tsunami & cross section & adults & $\begin{array}{l}\text { Hopkins Symptom } \\
\text { Checklist-25 }\end{array}$ & 1061 & $20.4 \%$ & 1 month & $38.6 \%$ & $15-90$ & 6 \\
\hline 17 & Chou & 2007 & China (taiwan) & Chi-Chi earthquake & cross section & adults & $\begin{array}{l}\text { Mini-international } \\
\text { Neuropsychiatric } \\
\text { Interview }\end{array}$ & 216 & $11.6 \%$ & $\begin{array}{l}6 \text { month, } 2 \\
\text { years, } 3 \text { years }\end{array}$ & $45.8 \%$ & $>=16$ & 6 \\
\hline
\end{tabular}


Table 1 General characteristic of the included studies with regard to risk factors for depression after natural disasters (Continued)

\begin{tabular}{|c|c|c|c|c|c|c|c|c|c|c|c|c|c|}
\hline$\overline{18}$ & Acierno & 2007 & France & Florida hurricane & cross section & adults & $\begin{array}{c}\text { Structured Clinical } \\
\text { Interview for DSM-IV }\end{array}$ & 1452 & $6.1 \%$ & 6-9 months & $48.1 \%$ & $>=18$ & 6 \\
\hline 19 & Chou & 2005 & Taiwan (china) & Chi-Chi earthquake & cross section & adults & $\begin{array}{l}\text { Mini-international } \\
\text { Neuropsychiatric } \\
\text { Interview }\end{array}$ & 442 & $9.5 \%$ & 4-6 months & $48.4 \%$ & $>=16$ & 7 \\
\hline 20 & Armenian & 2002 & Armenia & Armenian Earthquak & case control & adults & $\begin{array}{l}\text { Structured Clinical } \\
\text { Interview } \\
\text { for DSM-III-R }\end{array}$ & 760 & $52.0 \%$ & 2 year & $45.1 \%$ & $13-70$ & 9 \\
\hline 21 & Adams & 2014 & Armenia & Spring 2011 tornado & cross section & children & $\begin{array}{l}\text { National Survey of } \\
\text { Adolescents Depression } \\
\text { module for DSM-IV }\end{array}$ & 1514 & $7.5 \%$ & 8.8 months & $49.1 \%$ & $12-17$ & 8 \\
\hline 22 & Ye & 2014 & China & Wenchuan earthquake & cross section & children & $\begin{array}{l}\text { Depression Self-rating } \\
\text { Scale for Children }\end{array}$ & 1573 & $27.4 \%$ & 6 months & $45.8 \%$ & 15 (mean) & 7 \\
\hline 23 & Pan & 2013 & China & Wenchuan earthquake & cross section & children & $\begin{array}{l}\text { Zung self-rating } \\
\text { Dep11ression Scale }\end{array}$ & 362 & $44.8 \%$ & 3 years & $43.6 \%$ & $11-16$ & 8 \\
\hline 24 & Kadak & 2013 & Turkey & Van earthquake & cross section & children & $\begin{array}{l}\text { Child Depression } \\
\text { Inventory }\end{array}$ & 738 & $37.7 \%$ & 6 months & $55.0 \%$ & $13-17$ & 6 \\
\hline 25 & Wang & 2012 & China & Wenchuan earthquake & cross section & children & $\begin{array}{l}\text { Depression Self-rating } \\
\text { Scale for Children }\end{array}$ & 1841 & $19.5 \%$ & 10 months & $48.7 \%$ & $11-20$ & 7 \\
\hline 26 & Liu & 2011 & China & Wenchuan earthquake & cross section & children & $\begin{array}{l}\text { Trauma Symptom } \\
\text { Checklist for Children }\end{array}$ & 330 & $14.5 \%$ & $\begin{array}{l}6 \text { months and } \\
12 \text { months }\end{array}$ & $50.0 \%$ & $8-12$ & 4 \\
\hline 27 & Fan & 2011 & China & Wenchuan earthquake & cross section & children & $\begin{array}{l}\text { The Depression } \\
\text { Self-rating Scale } \\
\text { for Children }\end{array}$ & 2081 & $24.5 \%$ & 6 months & $45.9 \%$ & $\begin{array}{c}14.5 \\
\text { (mean) }\end{array}$ & 6 \\
\hline 28 & Lau & 2010 & China & Wenchuan earthquake & cross section & children & $\begin{array}{c}\text { Children's Depression } \\
\text { Inventory }\end{array}$ & 3324 & $22.6 \%$ & 1 month & $54.3 \%$ & $12-18$ & 7 \\
\hline 29 & Jia & 2010 & China & Wenchuan earthquake & cross section & children & $\begin{array}{c}\text { Children's Depression } \\
\text { Inventory }\end{array}$ & 596 & $13.9 \%$ & 15 months & $49.8 \%$ & $8-16$ & 7 \\
\hline 30 & Thienkrua & 2006 & Thailand & Thailand tsunami & cross section & children & $\begin{array}{l}\text { Birleson Depression } \\
\text { Self-Rating Scale }\end{array}$ & 371 & $8.4 \%$ & $\begin{array}{l}2 \text { months } \\
\text { and } \\
9 \text { months }\end{array}$ & $46.9 \%$ & $7-14$ & 6 \\
\hline 31 & Eksi & 2007 & Turkey & Turkey earthquake & cross section & children & $\begin{array}{l}\text { Structured Clinical } \\
\text { Interview for DSM-IV }\end{array}$ & 160 & $30.6 \%$ & 6-20 weeks & $36.3 \%$ & $9-18$ & 6 \\
\hline
\end{tabular}


Table 2 Risk Factors for depression after natural disasters in adults

\begin{tabular}{|c|c|c|c|c|c|c|c|c|c|c|c|c|c|}
\hline & \multicolumn{5}{|c|}{ All studies } & \multicolumn{2}{|c|}{ High quality } & \multicolumn{2}{|c|}{ Adjustment } & \multicolumn{2}{|c|}{ Exclude wenchuan } & \multicolumn{2}{|c|}{ Within 6 months } \\
\hline & $N$ & OR $(95 \% \mathrm{Cl})$ & $\mathrm{I}^{2}$ (P value) & Egger test & Trim and fill & N & OR $(95 \% \mathrm{Cl})$ & $N$ & OR $(95 \% \mathrm{Cl})$ & $N$ & OR $(95 \% \mathrm{Cl})$ & N & OR $(95 \% \mathrm{Cl})$ \\
\hline \multicolumn{14}{|l|}{ Basic characteristics } \\
\hline \multirow[t]{2}{*}{ Age (older) } & 13 & $1.03(0.70-1.51)$ & $80.8 \%$ & $P=0.839$ & - & 5 & $1.37(0.86-2.17)$ & 8 & $0.98(0.56-1.70)$ & 8 & $0.93(0.61-1.44)$ & 6 & $0.81(0.37-1.78)$ \\
\hline & & & $(p<0.001)$ & & & & & & & & & & \\
\hline \multirow[t]{2}{*}{ Gender (female) } & 18 & $1.57(1.39-1.79)$ & $18.5 \%$ & $p=0.282$ & - & 8 & $1.60(1.35-1.90)$ & 13 & $1.65(1.43-1.89)$ & 13 & $1.61(1.41-1.84)$ & 10 & $1.74(1.50-2.02)$ \\
\hline & & & $(p=0.233)$ & & & & & & & & & & \\
\hline \multirow[t]{2}{*}{ Education (lower) } & 14 & $1.70(1.29-2.23)$ & $72.7 \%$ & $P=0.008$ & $1.31(0.98-1.76)$ & 7 & $1.44(1.04-1.99)$ & 7 & $1.93(1.20-3.10)$ & 9 & $1.48(1.13-1.93)$ & 9 & $2.18(1.41-3.37)$ \\
\hline & & & $(p<0.001)$ & & & & & & & & & & \\
\hline \multirow[t]{2}{*}{ Marry (not) } & 12 & $1.43(1.03-1.98)$ & $73.7 \%$ & $P=0.030$ & $1.43(1.03-1.98)$ & 5 & $1.15(0.95-1.40)$ & 6 & $2.47(0.90-6.78)$ & 7 & $1.02(0.80-1.30)$ & 7 & $1.47(0.88-2.47)$ \\
\hline & & & $(p<0.001)$ & & & & & & & & & & \\
\hline \multirow[t]{2}{*}{ Religion (yes) } & 4 & $1.37(1.02-1.86)$ & $0 \%$ & $P=0.469$ & - & 2 & $1.51(1.07-2.13)$ & - & - & 2 & $1.25(0.74-2.11)$ & 2 & $1.25(0.74-2.11)$ \\
\hline & & & $(p=0.635)$ & & & & & & & & & & \\
\hline \multirow[t]{2}{*}{ Prior trauma (yes) } & 4 & $2.26(1.34-3.81)$ & $67.9 \%$ & $P=0.352$ & - & 1 & $1.50(0.80-2.60)$ & 2 & $1.73(1.33-2.25)$ & 4 & $1.73(1.33-2.25)$ & 2 & $2.85(0.90-8.96)$ \\
\hline & & & $(p=0.025)$ & & & & & & & & & & \\
\hline \multirow[t]{2}{*}{ Prior physical illness (yes) } & 5 & $1.71(0.91-3.20)$ & $81.3 \%$ & $P=0.175$ & - & 2 & $1.34(0.99-1.83)$ & 2 & $2.42(0.58-10.08)$ & 1 & $4.28(1.78-10.30)$ & 2 & $1.59(0.24-10.35)$ \\
\hline & & & $(p<0.001)$ & & & & & & & & & & \\
\hline \multicolumn{14}{|l|}{ Trauma characteristics } \\
\hline \multirow[t]{2}{*}{ Being trapped (yes) } & 2 & $1.01(0.60-1.69)$ & $0 \%$ & - & - & - & - & 1 & $0.94(0.54-1.62)$ & 1 & $0.94(0.54-1.62)$ & 2 & $1.01(0.60-1.69)$ \\
\hline & & & $(p=0.465)$ & & & & & & & & & & \\
\hline \multirow[t]{2}{*}{ Scare (yes) } & 2 & $1.58(1.37-1.83)$ & $0 \%$ & - & - & 2 & $1.58(1.37-1.83)$ & 2 & $1.58(1.37-1.83)$ & - & - & - & - \\
\hline & & & $(p=0.535)$ & & & & & & & & & & \\
\hline \multirow[t]{2}{*}{ Injure (yes) } & 5 & $1.69(1.39-2.06)$ & $0 \%$ & $P=0.559$ & - & 3 & $1.83(1.43-2.35)$ & 4 & $1.66(1.36-2.04)$ & 2 & $1.49(1.12-1.97)$ & 3 & $1.55(1.19-2.02)$ \\
\hline & & & $(p=0.655)$ & & & & & & & & & & \\
\hline \multirow[t]{2}{*}{ Witness injury/death (yes) } & 3 & $1.25(0.98-1.60)$ & $0 \%$ & $P=0.120$ & - & 2 & $1.32(0.92-1.88)$ & - & - & 2 & $1.32(0.92-1.88)$ & 2 & $1.23(0.93-1.64)$ \\
\hline & & & $(p=0.931)$ & & & & & & & & & & \\
\hline \multirow[t]{2}{*}{ Bereavement (yes) } & 6 & $1.51(1.22-1.86)$ & $0 \%$ & $P=0.454$ & - & 4 & $1.43(1.11-1.83)$ & 4 & $1.54(1.21-1.97)$ & 3 & $1.49(1.12-1.99)$ & 2 & $1.52(1.12-2.05)$ \\
\hline & & & $(p=0.906)$ & & & & & & & & & & \\
\hline \multicolumn{14}{|l|}{ Post-trauma characteristics } \\
\hline \multirow[t]{2}{*}{ Social support (yes) } & 8 & $0.95(0.90-1.01)$ & $90.1 \%$ & $P=0.014$ & $0.95(0.90-1.01)$ & 4 & $0.99(0.95-1.03)$ & 6 & $0.95(0.89-1.01)$ & 5 & $0.5(0.31-0.80)$ & 2 & $0.92(0.77-1.10)$ \\
\hline & & & $(p<0.001)$ & & & & & & & & & & \\
\hline \multirow[t]{2}{*}{ Employment (no) } & 9 & $1.55(1.02-2.37)$ & $89.4 \%$ & $P=0.703$ & - & 5 & $1.57(1.30-1.91)$ & 9 & $1.55(1.02-2.37)$ & 6 & $1.80(1.04-3.12)$ & 4 & $1.85(0.85-4.01)$ \\
\hline & & & $(p<0.001)$ & & & & & & & & & & \\
\hline \multirow[t]{2}{*}{ Loss of property (yes) } & 7 & $1.66(1.11-2.47)$ & $74.7 \%$ & $P=0.512$ & - & 5 & $1.32(0.92-1.91)$ & 3 & $2.02(0.92-4.43)$ & 5 & $1.91(1.15-3.20)$ & 3 & $2.14(1.01-4.54)$ \\
\hline & & & $(p=0.001)$ & & & & & & & & & & \\
\hline \multirow[t]{2}{*}{ House damage (yes) } & 8 & $1.40(1.00-1.88)$ & $51.3 \%$ & $P=0.308$ & - & 5 & $1.65(1.04-2.63)$ & 3 & $1.66(0.89-3.10)$ & 4 & $1.38(1.01-1.89)$ & 6 & $1.37(0.94-2.01)$ \\
\hline & & & $(p=0.045)$ & & & & & & & & & & \\
\hline
\end{tabular}




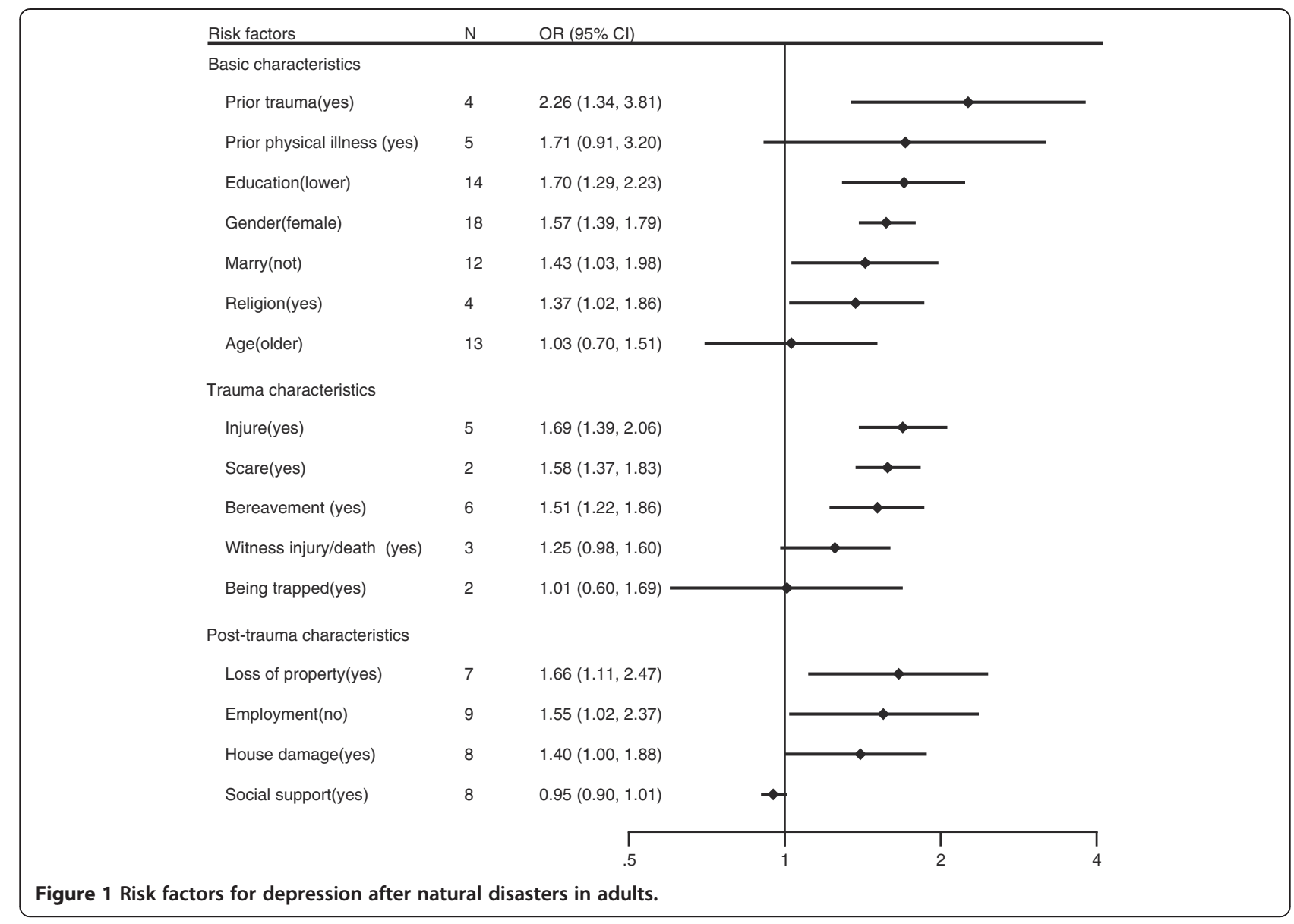

The initial analysis of the trauma characteristics of survivors (i.e. before excluding low-quality and unadjusted studies) revealed that all five factors were associated with risk of depression; the pooled ORs were 1.73 (95\% CI, 1.17-2.56) for being trapped, 2.39 (95\% CI, 1.50-3.82) for experiencing fear, 2.60 (95\% CI, 1.49-5.53) for experiencing injury, 1.68 (95\% CI, 1.33-2.10) for witnessing injury/death, and 2.85 (95\% CI, 1.59-5.11) for bereavement. However, after excluding low-quality and unadjusted studies, only two factors (bereavement and witnessing an injury or death) were still significantly associated with risk of depression.

Finally, with regard to the post-trauma characteristics of survivors, only one study found that social support was a protective factor for onset of depression (OR $0.21,95 \%$ CI, $0.15-0.28)$.

\section{Discussion}

To the best of our knowledge, this is the first meta-analysis focusing on risk factors for depression in populations specifically affected by natural disasters. Our synthesis of the relevant published English-language articles provided strong evidence for risk factors of depression following natural disasters. This study analyzed 31 published observational studies (1 case-control, 1 cohort, and 29 cross-sectional studies, including a total of 41,107 people). A total of 16 risk factors of depression in the survivors of natural disasters were explored in our study and categorized into three types: basic characteristics, trauma characteristics, and post-trauma characteristics.

With regard to basic characteristic, a common risk factor for the development of depression in both children and adults was prior exposure to trauma. This finding is consistent with previous research suggesting that the accumulation of violent traumatic events throughout the life course could increase the risk of depression [17,48]. Further common risk factors for adults were poor education, holding religious beliefs, and being female. Educational level indirectly influences economic resources, social status, social networks, health behaviour, and so on [49]. In natural disasters, people with higher education levels might use better coping methods because they have greater social resources, thus reducing the incidence of depression. Another interesting finding was that adults with religious beliefs were more susceptible to depression. Some research does substantiate our finding - for instance, Buddhists appeared to experience poorer mental health in the aftermath of the Wenchuan earthquake compared to people without religious beliefs [50]. This result might partly be explained by 
Table 3 Risk factors for depression after natural disasters in children

\begin{tabular}{|c|c|c|c|c|c|c|c|c|c|c|c|c|c|}
\hline & \multicolumn{5}{|c|}{ All studies } & \multicolumn{2}{|r|}{ High quality } & \multicolumn{2}{|r|}{ Adjustment } & \multicolumn{2}{|c|}{ Exclude wenchuan } & \multicolumn{2}{|c|}{ Within 6 months } \\
\hline & $N$ & OR (95\%cl) & $\begin{array}{c}1^{2} \\
\text { (P value) }\end{array}$ & Egger test & Trim and fill & $N$ & OR (95\%cl) & $\mathrm{N}$ & OR (95\%cl) & $\mathrm{N}$ & OR $(95 \% \mathrm{cl})$ & $\mathrm{N}$ & OR $(95 \% \mathrm{cl})$ \\
\hline \multicolumn{14}{|l|}{ Basic characteristics } \\
\hline \multirow[t]{2}{*}{ Age (older) } & 7 & $1.20(0.92-1.55)$ & $73.5 \%$ & $P=0.663$ & - & 2 & $1.01(0.67-1.52)$ & 6 & $1.60(0.88-1.53)$ & 3 & $1.10(0.75-1.62)$ & 4 & $1.21(0.84-1.74)$ \\
\hline & & & $(p=0.001)$ & & & & & & & & & & \\
\hline \multirow[t]{2}{*}{ Gender (female) } & 9 & $0.88(0.61-1.27)$ & $87.9 \%$ & $P=0.023$ & $0.88(0.61-1.27)$ & 4 & $0.94(0.50-1.76)$ & 7 & $0.92(0.61-1.39)$ & 4 & $0.51(0.26-0.99)$ & 4 & $0.83(0.44-1.56)$ \\
\hline & & & $(p<0.001)$ & & & & & & & & & & \\
\hline \multirow[t]{2}{*}{ Prior trauma (yes) } & 3 & $1.73(1.16-2.58)$ & $86.6 \%$ & $P=0.555-$ & - & 2 & $1.94(1.21-3.11)$ & 3 & $1.73(1.16-2.58)$ & 2 & $1.82(0.95-3.49)$ & 2 & $1.47(1.21-1.78)$ \\
\hline & & & $(p=0.001)$ & & & & & & & & & & \\
\hline \multicolumn{14}{|l|}{ Trauma characteristics } \\
\hline \multirow[t]{2}{*}{ Being trapped (yes) } & 3 & $1.73(1.17-2.56)$ & $0 \%$ & $P=0.218$ & - & 1 & $1.70(1.02-2.84)$ & 2 & $2.15(0.82-5.65)$ & 1 & $6.03(0.79-45.93)$ & 2 & $2.15(0.71-6.47)$ \\
\hline & & & $(p=0.464)$ & & & & & & & & & & \\
\hline \multirow[t]{2}{*}{ Scare (yes) } & 4 & $2.39(1.50-3.82)$ & $52.9 \%$ & $P=0.110$ & - & 2 & $2.20(0.10-4.88)$ & 2 & $2.20(0.10-4.88)$ & 1 & $2.73(1.02-7.29)$ & 1 & $3.07(1.47-6.40)$ \\
\hline & & & $(p=0.095)$ & & & & & & & & & & \\
\hline Injury (yes) & 5 & $2.60(1.49-5.53)$ & $49.1 \%(p=0.097)$ & $P=0.722$ & - & 2 & $2.69(0.69-10.44)$ & 3 & $3.01(1.17-7.72)$ & 3 & $3.08(1.23-7.73)$ & 2 & $3.54(1.99-6.30)$ \\
\hline \multirow[t]{2}{*}{ Witness injury/death (yes) } & 6 & $1.68(1.33-2.10)$ & $42.5 \%$ & $P=0.461$ & - & 3 & $1.43(1.00-2.04)$ & 4 & $1.46(1.09-1.94)$ & 2 & $2.11(1.15-3.86)$ & 2 & $2.48(1.46-4.21)$ \\
\hline & & & $(p=0.122)$ & & & & & & & & & & \\
\hline \multirow[t]{2}{*}{ Bereavement (yes) } & 6 & $2.85(1.59-5.11)$ & $74.7 \%$ & $P=0.014$ & $2.01(1.12-3.62)$ & 2 & $2.26(0.81-6.29)$ & 4 & $2.64(1.31-5.34)$ & 3 & $3.02(1.11-8.26)$ & 3 & $4.58(1.12-18.78)$ \\
\hline & & & $(p=0.001)$ & & & & & & & & & & \\
\hline \multicolumn{14}{|c|}{ Post-trauma characteristics } \\
\hline Social support (yes) & 1 & $0.21(0.15-0.28)$ & - & - & - & 1 & $0.21(0.15-0.28)$ & 1 & $0.21(0.15-0.28)$ & 1 & $0.21(0.15-0.28)$ & 1 & $0.21(0.15-0.28)$ \\
\hline \multirow[t]{2}{*}{ Loss of property (yes) } & 5 & $0.97(0.83-1.15)$ & $14.0 \%$ & $P=0.037$ & - & 3 & $0.92(0.79-1.06)$ & 5 & $0.97(0.83-1.15)$ & 2 & $1.25(0.54-2.89)$ & 1 & $1.02(0.71-1.47)$ \\
\hline & & & $(p=0.325)$ & & & & & & & & & & \\
\hline \multirow[t]{2}{*}{ House damage (yes) } & 5 & $1.05(0.84-1.32)$ & $0 \%$ & $P=0.141$ & - & 3 & $1.00(0.76-1.34)$ & 5 & $1.05(0.84-1.32)$ & 2 & $1.41(0.87-2.27)$ & 3 & $1.24(0.90-1.69)$ \\
\hline & & & $(p=0.500)$ & & & & & & & & & & \\
\hline
\end{tabular}


the belief that natural disasters are a punishment from God; this might lead to an increase in negative feelings such as guilt and depression [51].

Women were more likely to be depressed following a natural disaster than men were. Previous studies have indicated that women are more sensitive to threats, less likely to use effective coping strategies, and tend to interpret disasters more negatively than men do [9]. In addition, women are thought to be more sensitive to stress hormones, so their ability to manage stressful situations may be relatively poorer than men's ability [22]. We did not find any gender difference among children in this meta-analysis. This may partly be explained by the fact that gender-specific characteristics have not yet fully developed during childhood [23]. We also found that married people are comparatively less harmed by natural disasters than were people who were unmarried, divorced, or widowed. Information about formal relationship status may potentially identify individuals with limited support structures and associated risk, offering useful directions for mental health monitoring and outreach programs [3].

In terms of trauma characteristics, children and adults shared three risk factors: experiencing fear, injury, and bereavement. It is likely that fear per se does not increase risk of depression; this effect is perhaps mediated instead by subjective experience of a natural disaster and personality type [10]. For example, individuals with high neuroticism tend to be more reactive and sensitive to adverse events, possibly increasing their risk of developing depression. The link between being injured and depression is possibly related to the severity of the injuries; injuries after a natural disaster are often so severe that they result in amputation and disability [21]. The onset of disability is likely to reduce quality of life in some people, and this loss of quality of life might lead to depression. Other studies have also demonstrated this link between physical injury and depression. Injuries that influence emotional and behavioural well-being may particularly contribute to the onset of depression [52,53]. Finally, bereavement after natural disasters was a risk factor for depression in both adult and child samples. Previous studies have indicated that bereavement in childhood is a potential risk factor for subsequent psychopathology [54]; furthermore, the extent of loss of family members is highly correlated with the incidence of depression, especially for children [55].

Children were more likely to develop depression if they were trapped or witnessed injury or death during the disaster. Being trapped is likely to generate a traumatic memory directly associated with the negative event, which is considered an important precursor to depression. It is unsurprising that children who witnessed someone being njured or killed during a disaster may have experienced intense fear during this time; as described above, fear can itself be a predictor of depression.

With regard to post-trauma characteristics, lower levels of social support were associated with a higher risk of depression in children. Lack of care and support from others may foster feelings of inferiority and insecurity among children, which act as catalysts for the development of depression [56]. However, this relationship was not found among adults. Modes of thinking are more complex in 
adults and so social support may not be as effective in relieving their negative moods after natural disasters [11]. However, only one study included in the metaanalysis investigated the relationship between social support and depression in children, so it is difficult to make generalisations without further research on this issue.

Adults who were unemployed were more likely to exhibit depressive symptoms after a natural disaster. This suggests that a loss of resources results in people being unable to care for their families to the extent that they could before the disaster [17,57]. Loss of property and house damage were also potential predictive factors for adult depression, indicating the need to examine the effects of socioeconomic conditions after the disaster on depression.

There are several potential limitations to our metaanalysis. First, we included only observational studies, which can be prone to biases in sample selection, recall, and information evaluation, as well as confounding bias. Second, the majority of the studies included in the metaanalysis (21 studies; 67.7\%), were based on risk factors for depression specifically after an earthquake. Thus, it is premature to apply our results to survivors of all types of natural disasters. Finally, many of the variables included in the analysis were only examined in a small proportion of studies, which restricts the generalizability of the findings. Nevertheless, our study helps to highlight areas that would benefit from further investigation.

\section{Conclusions}

In conclusion, our study demonstrated several risk factors for depression in children and adults following natural disasters. Despite the methodological limitations of the studies that we included in the meta-analysis, these findings are valuable for understanding how to reduce symptoms of depression following a natural disaster. Such research may provide clear intervention directions and result in development of psychosocial support programs for at-risk groups, or assist in the development of prevention programs for depression. General practitioners should be aware of depressive symptoms, and careful consideration should be given to routine screens for depression during the reconstruction process following natural disasters. Further research will also be required to determine suitable interventions for improving the mental health conditions of survivors in areas affected by natural disasters. Above all, postdisaster mental health recovery programs that include early identification, on-going monitoring, preventive and intervention programs, and sustained psychosocial support are needed for the high-risk population of natural disaster survivors.

\section{Additional files}

Additional file 1: Moose statement - reporting checklist for authors, editors, and reviewers of meta-analyses of observational studies.

Additional file 2: Table S1. Electronic databases and search query.

Additional file 3: Figure S1. Search results and excluded/Included studies. Additional file 4: Table S2. Excluded studies and reasons for exclusion.

\section{Abbreviations}

NOS: Newcastle-ottawa scale; $A H R Q$ : Agency for healthcare research and quality; OR: Odds ratio; RR: Relative risk.

\section{Competing interests}

The authors declare that they have no competing interests.

\section{Authors' contributions}

$B T, X L$ and $L Z$ discussed and developed the question for this review. BT and $\mathrm{XL}$ carried out the searches. BT and XL assessed the eligibility of the studies for inclusion, extracted data and carried out all analysis. All authors were involved in interpreted and discussed results. BT wrote the first draft of this paper and it was reviewed by XL and LZ All authors agreed on the final draft of this study. LZ is the guarantor.

\section{Authors' information}

Bihan Tang and Xu Liu are co-first authors of this article.

\section{Acknowledgements}

Work on this manuscript was supported by the National Natural Science Foundation of China (Y. L., grant number 71103194, X. L., grant number 71303249, L. Z., grant number 91224005); and the Major Project of the "12th Five-Year Plan" of PLA (L. Z., grant number AWS12J002).

Received: 12 November 2013 Accepted: 13 June 2014 Published: 19 June 2014

\section{References}

1. Beaudoin CE: News, social capital and health in the context of Katrina. $J$ Health Care Poor Underserved 2007, 18(2):418-430.

2. Malhotra R, Chan A, Ostbye T: Prevalence and correlates of clinically significant depressive symptoms among elderly people in Sri Lanka: findings from a national survey. Int Psychogeriatr 2010, 22(2):227-236.

3. Cheng Z, Ma N, Yang L, Agho K, Stevens G, Raphael B, Cui L, Liu Y, Yan B, $\mathrm{Ma} H, \mathrm{Yu} X$ : Depression and posttraumatic stress disorder in temporary settlement residents 1 year after the sichuan earthquake. Asia-Pac J Public Health 2013, Published online May 10. doi:10.1177/1010539513485971.

4. Tracy M, Norris FH, Galea S: Differences in the determinants of posttraumatic stress disorder and depression after a mass traumatic event. Depress Anxiety 2011, 28(8):666-675.

5. Chou FH, Wu HC, Chou P, Su CY, Tsai KY, Chao SS, Chen MC, Su TT, Sun WJ, Ou-Yang WC: Epidemiologic psychiatric studies on post-disaster impact among Chi-Chi earthquake survivors in Yu-Chi, Taiwan. Psychiatry Clin Neurosci 2007, 61(4):370-378.

6. Jia Z, Tian W, He X, Liu W, Jin C, Ding H: Mental health and quality of life survey among child survivors of the 2008 Sichuan earthquake. Qual Life Res 2010, 19(9):1381-1391.

7. Van Griensven F, Chakkraband MLS, Thienkrua W, Pengjuntr W, Lopes Cardozo B, Tantipiwatanaskul P, Mock PA, Ekassawin S, Varangrat A, Gotway C, Sabin M, Tappero JW, Thailand Post-Tsunami Mental Health Study Group: Mental health problems among adults in tsunami-affected areas in southern Thailand. JAMA 2006, 296(5):537-548.

8. Liu M, Wang L, Shi Z, Zhang Z, Zhang K, Shen J: Mental health problems among children one-year after Sichuan earthquake in China: a follow-up study. PLoS One 2011, 6(2):e14706.

9. Zhang Z, Wang WZ, Shi ZB, Wang L, Zhang JX: Mental Health Problems among the Survivors in the hard-hit areas of the yushu earthquake. PLoS One 2012, 7(10):e46449.

10. Zhang Z, Shi Z, Wang L, Liu M: One year later: mental health problems among survivors in hard-hit areas of the Wenchuan earthquake. Public Health 2011, 125(5):293-300. 
11. Zhang Z, Shi Z, Wang L, Liu M: Post-traumatic stress disorder, anxiety and depression among the elderly: a survey of the hard-hit areas a year after the wenchuan earthquake. Stress Health 2012, 28(1):61-68.

12. Amstadter AB, Acierno R, Richardson LK, Kilpatrick DG, Gros DF, Gaboury MT, Tran TL, Trung LT, Tam NT, Tuan T, Buoi LT, Ha TT, Thach TD, Galea S: Posttyphoon prevalence of posttraumatic stress disorder, major depressive disorder, panic disorder, and generalized anxiety disorder in a Vietnamese sample. J Trauma Stress 2009, 22(3):180-188.

13. Wang W, Fu W, Wu J, Ma X, Sun X, Huang Y, Hashimoto K, Gao C: Prevalence of ptsd and depression among junior middle school students in a rural town far from the epicenter of the Wenchuan earthquake in China. PLoS One 2012, 7(7):e41665.

14. Lau JT, Yu X, Zhang J, Mak WW, Choi KC, Lui WW, Chan EY: Psychological distress among adolescents in Chengdu, Sichuan at 1 month after the 2008 Sichuan earthquake. Journal Urban Health 2010, 87(3):504-523.

15. Acierno R, Ruggiero KJ, Galea S, Resnick HS, Koenen K, Roitzsch J, de Arellano M, Boyle J, Kilpatrick DG: Psychological sequelae resulting from the 2004 Florida hurricanes: implications for postdisaster intervention. Am J Public Health 2007, 97(Suppl 1):S103-S108.

16. Gigantesco A, Mirante N, Granchelli C, Diodati G, Cofini V, Mancini C, Carbonelli A, Tarolla E, Minardi V, Salmaso S, D'Argenio P: Psychopathological chronic sequelae of the 2009 earthquake in L'Aquila, Italy. J Affect Disord 2013, 148(2-3):265-271.

17. Cerda M, Paczkowski M, Galea S, Nemethy K, Pean C, Desvarieux M: Psychopathology in the aftermath of the haiti earthquake: a populationbased study of posttraumatic stress disorder and major depression. Depress Anxiety 2013, 30(5):413-424.

18. Paranjothy S, Gallacher J, Amlot R, Rubin GJ, Page L, Baxter T, Wight J, Kirrage D, McNaught R, Palmer SR: Psychosocial impact of the summer 2007 floods in England. BMC Public Health 2011, 11:145.

19. Anwar J, Mpofu E, Matthews LR, Shadoul AF, Brock KE: Reproductive health and access to healthcare facilities: risk factors for depression and anxiety in women with an earthquake experience. BMC Public Health 2011, 11:523.

20. Armenian HK, Morikawa M, Melkonian AK, Hovanesian A, Akiskal K, Akiskal HS: Risk factors for depression in the survivors of the 1988 earthquake in Armenia. J Urban Health 2002, 79(3):373-382.

21. Eksi A, Braun KL, Ertem-Vehid H, Peykerli G, Saydam R, Toparlak D, Alyanak B: Risk factors for the development of PTSD and depression among child and adolescent victims following a 7.4 magnitude earthquake. Int $J$ Psychiatry Clin Pract 2007, 11(3):190-199.

22. Zhou X, Kang L, Sun X, Song H, Mao W, Huang X, Zhang Y, Li J: Risk factors of mental illness among adult survivors after the Wenchuan earthquake. Soc Psychiatry Psychiatr Epidemiol 2013, 48(6):907-915.

23. Kadak MT, Nasiroglu S, Boysan M, Aydin A: Risk factors predicting posttraumatic stress reactions in adolescents after 2011 Van earthquake. Compr Psychiatry 2013, 54(7):982-990.

24. Thienkrua W, Cardozo BL, Chakkraband ML, Guadamuz TE, Pengjuntr W, Tantipiwatanaskul P, Sakornsatian S, Ekassawin S, Panyayong B, Varangrat A Tappero JW, Schreiber M, van Griensven F, Thailand Post-Tsunami Mental Health Study Group: Symptoms of posttraumatic stress disorder and depression among children in tsunami-affected areas in southern Thailand. JAMA 2006, 296(5):549-559.

25. Fan F, Zhang Y, Yang Y, Mo L, Liu X: Symptoms of posttraumatic stress disorder, depression, and anxiety among adolescents following the 2008 Wenchuan earthquake in China. J Trauma Stress 2011, 24(1):44-53.

26. Pan X, Liu W, Deng G, Liu T, Yan J, Tang Y, Dong W, Cui Y, Xu M: Symptoms of posttraumatic stress disorder, depression, and anxiety among junior high school students in worst-hit areas 3 years after the wenchuan earthquake in China. Asia Pac J Public Health 2013,

27. Boscarino JA, Hoffman SN, Kirchner HL, Erlich PM, Adams RE, Figley CR, Solhkhah R: Mental health outcomes at the Jersey shore after hurricane Sandy. Int J Emerg Ment Health 2013, 15(3):147-158.

28. $\mathrm{Vu} \mathrm{L}$, Vanlandingham MJ: Physical and mental health consequences of Katrina on Vietnamese immigrants in New Orleans: a pre- and postdisaster assessment. J Immigr Minor Health 2012, 14(3):386-394.

29. Adams ZW, Sumner JA, Danielson CK, McCauley JL, Resnick HS, Gros K, Paul LA Welsh KE, Ruggiero KJ: Prevalence and predictors of PTSD and depression among adolescent victims of the Spring 2011 tornado outbreak. J Child Psychol Psychiatry 2014, Published online Feb 28. DOl: 10.1111/jcpp.12220.

30. Guo S, Tian D, Wang X, Xiao Y, He H, Qu Z, Zhang X: Protective effects of social support content and support source on depression and its prevalence 6 months after wenchuan earthquake. Stress Health 2014, Published online Mar 11. DOI: 10.1002/smi.2563.

31. Chou FHC, Su TTP, Chou P, Ou-Yang WC, Lu MK, Chien IC: Survey of psychiatric disorders in a Taiwanese village population six months after a major earthquake. J Formos Med Assoc 2005, 104(5):308-317.

32. Ye Y, Fan F, Li L, Han Q: Trajectory and predictors of depressive symptoms among adolescent survivors following the Wenchuan earthquake in China: a cohort study. Soc Psychiatry Psychiatr Epidemiol 2014, 49(6):943-952.

33. Nillni YI, Nosen E, Williams PA, Tracy M, Coffey SF, Galea S: Unique and related predictors of major depressive disorder, posttraumatic stress disorder, and their comorbidity after hurricane katrina. J Nerv Ment Dis 2013, 201(10):841-847.

34. Fichter MM, Kohlboeck G, Quadflieg N: The upper Bavarian longitudinal community study 1975-2004. 2. Long-term course and outcome of depression. A controlled study. Eur Arch Psychiatry Clin Neurosci 2008, 258(8):476-488

35. Rhebergen D, Batelaan NM, de Graaf R, Nolen WA, Spijker J, Beekman AT, Penninx BW: The 7-year course of depression and anxiety in the general population. Acta Psychiatr Scand 2011, 123(4):297-306.

36. Porter M, Haslam N: Predisplacement and postdisplacement factors associated with mental health of refugees and internally displaced persons: a meta-analysis. JAMA 2005, 294(5):602-612.

37. Steel Z, Chey T, Silove D, Marnane C, Bryant RA, van Ommeren M: Association of torture and other potentially traumatic events with mental health outcomes among populations exposed to mass conflict and displacement: a systematic review and meta-analysis. JAMA 2009, 302(5):537-549.

38. Pinquart $M$, Duberstein PR: Depression and cancer mortality: a meta-analysis. Psychol Med 2010, 40(11):1797-1810.

39. Bartoli F, Lillia N, Lax A, Crocamo C, Mantero V, Carra G, Agostoni E, Clerici M: Depression after stroke and risk of mortality: a systematic review and meta-analysis. Stroke Res Treat 2013, 2013:862978.

40. Stroup DF, Berlin JA, Morton SC, Olkin I, Williamson GD, Rennie D, Moher D, Becker BJ, Sipe TA, Thacker SB: Meta-analysis of observational studies in epidemiology: a proposal for reporting. meta-analysis of observational studies in epidemiology (MOOSE) group. JAMA 2000, 283(15):2008-2012.

41. Celiac Disease. Evidence Reports/Technology Assessments, No. 104 (Appendix D) http://www.ncbi.nlm.nih.gov/books/NBK35156.

42. Wells G, Shea B, O'connell D, Peterson J, Welch V, Losos M, Tugwell P: The Newcastle-Ottawa Scale (NOS) For Assessing The Quality Of Nonrandomised Studies In Meta-Analyses. In 3rd Symposium on Systematic Reviews: Beyond the Basics; 2000:3-5.

43. Salcioglu E, Basoglu M, Livanou M: Long-term psychological outcome for non-treatment-seeking earthquake survivors in Turkey. J Nerv Ment Dis 2003, 191(3):154-160.

44. Jia Z, Tian W, Liu W, Cao Y, Yan J, Shun Z: Are the elderly more vulnerable to psychological impact of natural disaster? A population-based survey of adult survivors of the 2008 Sichuan earthquake. BMC Public Health 2010, 10:172.

45. DerSimonian R, Laird N: Meta-analysis in clinical trials. Control Clin Trials 1986, 7(3):177-188

46. Higgins JP, Thompson SG, Deeks JJ, Altman DG: Measuring inconsistency in meta-analyses. BMJ 2003, 327(7414):557-560.

47. Egger M, Davey Smith G, Schneider M, Minder C: Bias in meta-analysis detected by a simple, graphical test. BMJ 1997, 315(7109):629-634.

48. Bremner JD, Southwick S, Brett E, Fontana A, Rosenheck R, Charney DS: Dissociation and posttraumatic stress disorder in Vietnam combat veterans. Am J Psychiatry 1992, 149(3):328-332.

49. Lee J: Pathways from education to depression. J Cross Cult Gerontol 2011, 26(2):121-135

50. Wang T, Han B: Buddhism belief and mental health status of aging survivors in Wenchuan earthquake disaster area. Chin J Gerontol 2009, 29:1272-1275

51. Chen $Y Y$, Koenig HG: Traumatic stress and religion: Is there a relationship? A review of empirical findings. J Relig Health 2006, 45(3):371-381

52. Stoddard FJ, Saxe G: Ten-year research review of physical injuries. J Am Acad Child Adolesc Psychiatry 2001, 40(10):1128-1145.

53. Vehid HE, Alyanak B, Eksi A: Suicide ideation after the 1999 earthquake in Marmara, Turkey. Tohoku J Exp Med 2006, 208(1):19-24. 
54. Weller RA, Weller EB, Fristad MA, Bowes JM: Depression in recently bereaved prepubertal children. Am J Psychiatry 1991, 148(11):1536-1540.

55. Goenjian AK, Pynoos RS, Steinberg AM, Najarian LM, Asarnow JR, Karayan I, Ghurabi M, Fairbanks LA: Psychiatric comorbidity in children after the 1988 earthquake in Armenia. J Am Acad Child Adolesc Psychiatry 1995, 34(9):1174-1184.

56. Sajjadi H, Mohaqeqi Kamal SH, Rafiey H, Vameghi M, Forouzan AS, Rezaei M: A systematic review of the prevalence and risk factors of depression among iranian adolescents. Glob J Health Sci 2013, 5(3):16-27.

57. Norris FH, Friedman MJ, Watson PJ, Byrne CM, Diaz E, Kaniasty K: 60,000 disaster victims speak: part I. An empirical review of the empirical literature, 1981-2001. Psychiatry 2002, 65(3):207-239.

doi:10.1186/1471-2458-14-623

Cite this article as: Tang et al: A meta-analysis of risk factors for depression in adults and children after natural disasters. BMC Public Health 2014 14:623.

\section{Submit your next manuscript to BioMed Central and take full advantage of:}

- Convenient online submission

- Thorough peer review

- No space constraints or color figure charges

- Immediate publication on acceptance

- Inclusion in PubMed, CAS, Scopus and Google Scholar

- Research which is freely available for redistribution 